\title{
The American Academy of Family Physician's Approach to Developing and Supporting the Intrinsically Motivated Learner
}

\author{
Clif Knight, MD, CPE, FAAFP
}

The American Academy of Family Physicians has 72,600 active members and a robust continuing medical education platform. A foundational principle of their work is an assumption of an intrinsic motivation of physicians to learn in order to better take care of their patients and communities. This commentary presents their vision of individualized learning portfolio in which individual family physicians utilize data to identify their gaps of knowledge and performance over time and use a varirty of CME, including performance improvement, to address and lessen those gaps, with the Academy serving as a navigator and facilitator, all while supporting intrinsic motivation to learn and improve. (J Am Board Fam Med 2020;33:S21-S23.)

Keywords: Academies and Institutes, Continuing Medical Education, Family Physicians, Learning, Motivation

Continuous professional development has been valued by family medicine's specialty society from the very beginning. In 1947, the American Academy of General Practice (AAGP) formed with membership requirements, including a continuing medical education (CME) achievement. This was a deliberate and tangible, self-imposed commitment to professionalism and should not be overlooked as an exemplar. This requirement was a proud differentiator for general practitioners. At that time, the first CME credit system was developed by the AAGP. As the AAGP became the American Academy of Family Physicians (AAFP) and the specialty of family practice was chartered with the advent of the American Board of Family Practice in 1969, our CME requirement of 150 hours during each 3 -year cycle persisted. The AAFP's CME credit system remains the only specialty-specific CME credit system and is 1 of only 3 in the United States (the others being those of the American Medical Association [AMA] and the American Osteopathic Association). In addition, the

This article was externally peer reviewed.

Submitted 20 November 2019; revised 14 May 2020; accepted 19 May 2020.

From the Senior Vice President for Education, American Academy of Family Physicians, Leawood, KS.

Funding: None.

Conflict of interest: Employee of the AAFP.

Corresponding author: Clif Knight, MD, CPE, FAAFP, 11400 Tomahawk Creek Parkway, Leawood, KS 66211 (E-mail: cknight@aafp.org).
AAFP is 1 of very few specialty societies that requires CME for membership-now 150 credits (rather than hours) in each 3-year cycle. Family medicine's enduring commitment to CME sets the benchmark for medical specialties.

At the completion of formal training, no physician has met their full professional potential. Just as medical knowledge base expands continuously, so must the knowledge base, skill sets, and competence of a family physician. In an effort to continuously improve professional acumen, it seems family physicians are often intrinsically motivated to learn. Based on information gathered from AAFP members, they seek formal CME activities that are relevant to their practice, seen as a valuable use of time, address a perceived knowledge gap, and ultimately improve care they provide. A reasonable goal is to encourage acting on this intrinsic motivation by awarding CME credit and American Board of Family Medicine (ABFM) Family Medicine Certification points to reinforce completing the desired learning activities. Receiving credit for meaningful activities is an important aspect for family physician learnersespecially if accomplished in a way that minimizes administrative burden.

In the mid 2000s, a new category of CME credit was created by the AMA to recognize physicians not only for participating in formal learning activities but also for demonstrating efforts to reflect on their care of patients and implement improvements 
in care delivery as a result. This novel approach to $\mathrm{CME}$, Performance Improvement CME (PI$\mathrm{CME}$ ), incentivizes venturing beyond passive learning to a commitment to changing practice and then studying the effect of the change. Importantly, this also decouples credit awarded simply from time (CME hours) to recognize process (CME credits). This incorporates a 3 -stage approach of measuring and recognizing a practice gap, implementing a change for improvement, and subsequently studying the results of that change. Early examples include the AAFP's METRIC program and the ABFM's practice improvement modules as a component of maintenance of certification (MOC). Although well intended and thoughtfully developed, versions of these early offerings were often seen by learners as more box-checking exercises with extensive data-entry burden rather than being truly practice relevant and impactful on quality of care. The AAFP recognized an attrition rate higher than anticipated and desired in their METRIC activities. I label these efforts as "PI-CME 1.0."

The evolution of PI-CME at the AAFP has led to a more comprehensive PerformanceNavigator (PN) approach to PI-CME activities. PN consists of multiple stages of learner activities, including baseline practice assessment, intensive interactive learning sessions to bolster the learner's knowledge base, development of an individualized action plan, application of the plan, postimplementation reassessment of practice, and finally reflection on results to drive the development of further improvement interventions. This PI-CME activity meets ABFM MOC performance improvement (PI) requirements. Importantly, the AAFP has found that participants in PN report a higher rate of commitment to practice change than that seen in any other category of AAFP CME activities-over $90 \%$. The significant resources embedded in PN support dedicated, intrinsically motivated learners in their desire to improve the care they provide, which is supported by social learning theoryphysician-to-physician interaction through embedded learning communities. Concurrent to the AAFP's development of PN, the ABFM now offers a broader array of PI activity options, including a selfdirected PI activity option that provides credit for quality-improvement activities that physicians are already doing in practice, as well as new activities that can be uniquely tailored for any family physician, regardless of practice scope and setting. I call this current state "PI-CME 2.0".
AAFP member survey data indicate that approximately $80 \%$ of family physicians plan to implement some sort of performance improvement in the next year. Despite our best efforts to date, the uptake of PI-CME remains relatively minor compared with other categories of $\mathrm{CME}$. AAFP data reveal that PI-CME accounts for $10 \%$ or less of all CME credit reported by family physicians over the past 4 years. This is somewhat a reflection on the fact that less than $1 \%$ of CME activity credit applications include PI-CME. The disconnect between an apparent commitment to performance improvement and actual reporting of participation in formal PICME activities presents an opportunity to better acknowledge and reward practice enhancement efforts. Building on the construct of PN, the AAFP envisions tapping even deeper into family physicians' intrinsic desire and motivation to continuously improve the care they provide and more importantly the health outcomes of the patients, families, and communities they serve.

What will "PI-CME 3.0" look like? A provocative concept we propose is to consider is a "Meta PI-CME" approach. This is simply continuous overall cycles of gathering data from multiple sources to inform one's self about their current state (we know physicians' self-assessment of their knowledge gaps alone is woefully inaccurate); designing an individualized educational plan and using that plan to participate in learning activities, including PICME; implementing practice changes based on learning (new knowledge and new skills); and then starting the next cycle with reassessment. This follows the classic Plan-Do-Study-Act (PDSA) cycle of improvements repeatedly and frequently throughout a family physician's career rather than " 1 and done" or sporadically.

AAFP surveys reveal that $89 \%$ of family physicians have interest in the AAFP offering an assessment tool to help determine detailed and specific medical knowledge gaps across all body systems. Assessment information should include practice-level statistics regarding process and outcome measures extending beyond traditional medical knowledge into other competencies, including communication, team-based care, and professionalism. Data generated through ABFM MOC activities, including Continuous Knowledge Self-Assessment (CKSA) and Family Medicine Certification Longitudinal Assessment (FMCLA), could be a valuable component of needs assessment composition. 
How could we better encourage a broad adoption of the Meta PI-CME approach? The AAFP is considering a strategy of providing CME credit for completing a formal process of needs assessment, development of an individualized educational plan, acting on the plan, and following up with reassessment. By receiving CME credit for this planning activity, the intrinsically motivated learner, seeking to improve the care they provide and health outcomes of their population served, would be rewarded for completion of this formal practice.

The AAFP's vision for lifelong learning is summed in a strategic objective and correlating strategy statement prioritized by the AAFP Board of Directors in 2019: "The AAFP will equip members with clinical expertise to improve individual and population health. The AAFP will provide broad scope, personalized learning opportunities for members and other learners through relevant educational venues and formats."

The AAFP is working to achieve personalized learning support to provide family physicians with relevant, practice-enhancing activities. CME should promote that family physicians maintain a broad scope of clinical practice. Continuous engagement in $\mathrm{CME}$ will be enhanced by opportunities to more frequently participate in CME activities and effectively use focused learning communities. Formats and venues for the delivery of CME are expanding, soon to include more virtual and online video and audio options in addition to traditional, and still very popular, live in-person events.

Family physicians have a rich and robust history of dedication to continuous, lifelong learning. A challenge to consider is how we may best set out to transform the many extrinsic motivators in a physicians' life (i.e., reimbursement and pay for quality, hospital system-driven quality improvements, and licensure requirements) to align them with and potentiate intrinsic motivation. As a developer of CME activities and the national CME Credit System, the AAFP aims to encourage, support, and indeed reward learners for exhibiting behaviors reflecting their dedication-this manifestation of intrinsically motivated learning.

To see this article online, please go to: http://jabfm.org/content/ 33/Supplement/S21.full. 